\title{
Concurrent infection with Talaromyces marneffei and Cryptococcus neoformans in a patient without HIV infection
}

\author{
SUSU HE, DONGQING LV, YOUZU XU, XIAOMAI WU and LING LIN \\ Department of Respiratory Medicine, Taizhou Hospital of Wenzhou Medical University, Linhai, Zhejiang 317000, P.R. China
}

Received October 5, 2018; Accepted July 29, 2019

DOI: $10.3892 / \mathrm{etm} .2019 .8172$

\begin{abstract}
A case report of coinfection with Talaromyces marneffei (T. marneffei) and Cryptococcus neoformans (C. neoformans) is presented in a 57-year-old woman with hemolytic anemia who received dexamethasone for 8 years. To the best of our knowledge, this patient was successfully treated with voriconazole. This is the first case of T. marneffei and C. neoformans coinfection in a HIV-negative host. Clinicians should be aware of concomitant infection with T. marneffe $i$ and other pathogens in immunocompromised hosts. The current case report highlights the importance of clinician awareness of concurrent infections with T. marneffe $i$ and other pathogens in immunosuppressed patients.
\end{abstract}

\section{Introduction}

Talaromyces marneffei (T. marneffei) is a rare pathogenic Talaromyces species in humans and is the only temperature-dependent dimorphic fungus of the Talaromyces genus. In 1973, DiSalvo et al (1) reported the first case of a natural T. marneffei infection in humans. Currently, T. marneffei infection is a common opportunistic infection in patients with HIV and is prevalent in South Asian countries and South China (2-7). In the past two decades, the incidence of Talaromyces spp. infection has risen dramatically in tandem with the HIV epidemic. T. marneffei infections generally occur in patients with late-stage acquired immune deficiency syndrome (AIDS) or immunodeficiency (2). Therefore, Talaromyces spp. infection is more likely to occur concurrently with other opportunistic infections (7-9). The simultaneous identification of T. marneffei and Cryptococcus neoformans (C. neoformans) from blood and bronchial mucosal biopsy cultures is uncommon, and very few cases have been reported to date (7). The diagnosis and treatment of concurrent

Correspondence to: Dr Ling Lin, Department of Respiratory Medicine, Taizhou Hospital of Wenzhou Medical University, 150 Ximen Street, Linhai, Zhejiang 317000, P.R. China

E-mail: linling@enzemed.com

Key words: talaromyces marneffei, Cryptococcus neoformans, immunosuppression, antifungal therapy, coinfection infection with $T$. marneffei and $C$. neoformansis challenging in the clinical setting (7). In the current case report, a concurrent infection with T. marneffei and $C$. neoformans (based on blood and bronchial mucosal biopsy cultures) is presented in a Chinese patient without HIV infection, which was successfully treated. A literature review was performed to provide new insight into the treatment of this rare concurrent infection.

\section{Case report}

In January 2017, a 57-year-old Chinese woman presented with a fever (maximum temperature, $39^{\circ} \mathrm{C}$ ), cough (coughing a moderate quantity of white sputum) and pharyngalgia, which began two days prior to hospital admission (12th January 2017; Taizhou Hospital of Wenzhou Medical University, China). The patient was diagnosed with hemolytic anemia 8 years prior to admission, and treated with dexamethasone $9 \mathrm{mg} / \mathrm{d}$, which was gradually reduced to $2.25 \mathrm{mg} / \mathrm{d}$ after symptoms improved. The patient was still taking dexamethasone at the time of admission. However, the patient could not provide any other details about Dexamethasone treatment. The patient had no recent or direct contact with specific plants including rotten sugar canes or animals such as bamboo rats and had not traveled to any endemic areas such as South Asian countries and South China. The CT scan performed at Xianju County People's Hospital (China) revealed the presence of a hyperdense mass in the right lower lung (Fig. 1). The patient was subsequently treated with antibiotics but exhibited a poor response.

The patient was then referred to Taizhou Hospital of Wenzhou Medical University, China on 12th January 2017 with a body temperature of $37.3^{\circ} \mathrm{C}$, which increased thereafter, a pulse rate of 115 beats $/ \mathrm{min}$, a respiratory rate of 17 breaths/min, a blood pressure of $107 / 94 \mathrm{mmHg}$ and an oxygen saturation of $99 \%$. A physical examination was performed upon admission and demonstrated palpable lymph nodes that were $1-3 \mathrm{~cm}$ in size, and tenderness over the left cervical and supraclavicular areas. On the second day of hospital admission, laboratory tests using whole blood specimen (BC-6800 plus; Mindray Medical International Limited) at $25^{\circ} \mathrm{C}$ for $1 \mathrm{~min}$ revealed a white blood cell count of 3,300 cells $/ \mathrm{ml}$ (normal range, $4.0-10 \times 10^{9}$ cells $/ 1$ ), neutrophils $93.3 \%$ (normal range, 40-75\%), lymphocytecount 200 cells $/ \mathrm{ml}$ (normal range, $1.1-3.2 \times 10^{9}$ cells/l), hemoglobin $61 \mathrm{~g} / \mathrm{dl}$ (normal range, $115-150 \mathrm{~g} / \mathrm{l}$ ) and platelet count $22,000 \mathrm{cells} / \mathrm{ml}$ (normal ranges: $125-350 \times 10^{9}$ cells/l). C-reactive protein level 
(CRP-M100; Mindray Medical International Limited) was $311 \mathrm{mg} / \mathrm{dl}$ (normal range, <5.0 mg/l) using whole blood specimen at $25^{\circ} \mathrm{C}$ for $2 \mathrm{~min}$ and a negative result for HIV antibodies (Microelisa Stripplate; Bejing Wantai Biological) using serum at $37^{\circ} \mathrm{C}$ for 120 min was determined. Serum chemistry (Beckman AU5800) using serum at $37^{\circ} \mathrm{C}$ for $40 \mathrm{~min}$ revealed that alanine transaminases was $22 \mathrm{U} / 1$ (normal range: 7-40 U/1) and aspartate transaminases was $32 \mathrm{U} / 1$ (normal range: 13-35 U/1), these levels were normal. A type-B ultrasound examination revealed left cervical, left supraclavicular, bilateral inguinal and retroperitoneal lymphadenopathy. Since the chest $\mathrm{CT}$ scan failed to reveal any enlarged pulmonary hilar or mediastinal lymph nodes, the patient did not undergo an endobronchial ultrasound bronchoscopy.

The patient was treated with Ceftazidime ( $2.0 \mathrm{~g}$ intravenous infusion per $12 \mathrm{~h}$ ) and Levofloxacin $(0.5 \mathrm{~g}$ iv infusion once a day) on the day of admission. On day 5 of treatment, hyperpyrexia occurred again similar to two days before admission and blood cultures were subsequently performed. Endoscopic esophageal ultrasound-guided fine-needle aspiration of the left supraclavicular lymph nodes was performed and the aspirates were submitted for histopathology. Blood cultures grew T. marneffe $i$ after 5 days of incubation at $25^{\circ} \mathrm{C}$, and the aspirate from a supraclavicular lymph node stained with Gomeri methenamine silver $\left(25^{\circ} \mathrm{C}\right.$ constant temperature water bath for $50 \mathrm{~min}$ ) revealed yeast-like fungi with transverse septa, which confirmed the presence of T. marneffei (Fig. 2). Subsequently, on hospital day 11, treatment with intravenous voriconazole $(6 \mathrm{mg} / \mathrm{kg}$ per $12 \mathrm{~h}$ for the first $24 \mathrm{~h}$, followed by $4 \mathrm{mg} / \mathrm{kg}$ per $12 \mathrm{~h})$ began. The fever subsided after 4 days of treatment, and the patients' cough and pharyngalgia also improved. The patient was treated with intravenous voriconazole for 2 weeks, and received oral voriconazole therapy of $200 \mathrm{mg}$ twice a day. Blood cultures were performed on hospital admission day 23 for the detection of T. marneffei. Blood cultures on day 5 did not exhibit T. marneffei growth, but did exhibit $C$. neoformans. Antimicrobial susceptibility testing of $C$. neoformans using the broth dilution method (10) (bioMerieux, Ltd.) was conducted and the rank order of potency, which was based on minimum inhibitory concentration (MIC) values, were itraconazole (0.06), voriconazole (0.125), amphotericin B (0.5), fluconazole (1) and 5-fluorocytosine (4). A period of 2 weeks into treatment with oral voriconazole, the patient underwent a CT scan of the chest on hospital day 37, which revealed deterioration compared with the previous scans (Fig. 3). On the same day, a bronchoscopy was performed, which revealed hyperplasia and edema of the airway mucosa of the right lower lobe bronchus (Fig. 4). Although the patient's symptoms had been significantly relieved, on day 38 , the patient was treated with intravenous voriconazole of $4 \mathrm{mg} / \mathrm{kg}$ per $12 \mathrm{~h}$ for 10 additional days based on the chest CT scan and bronchoscopy results. A period of 3 days later, the periodic acid-Schiff-stained $\left(25^{\circ} \mathrm{C} ; 15 \mathrm{~min}\right)$ preparation of bronchial mucosal mesenchyme confirmed the presence of $C$. neoformans (Fig. 5). The patient achieved dramatic clinical improvement and was discharged home on oral voriconazole (200 $\mathrm{mg}$ twice a day) for a period of 2 months. At the 2 month follow up following the completion of therapy, there was no evidence of ongoing infection and a repeat $\mathrm{CT}$ scan of the chest revealed significant improvement in the right lower lung lesion (Fig. 6).

\section{Discussion}

T. marneffei causes a disseminated and lethal fungal disease, which is known as talaromycosis. In South Asian countries, talaromycosis has become the third most common disease in patients infected with HIV, following tuberculosis and cryptococcosis (2-5). Talaromycosis is becoming increasingly prevalent in patients who are HIV-negative and exhibit no apparent risk factors or underlying immunodeficiency, including organ transplant recipients, patients with hematologic malignancies or patients receiving glucocorticoids or immunosuppressants (1,5,11-14). The majority of T. marneffei infections occur in patients with AIDS or an immunodeficiency disorder, whose CD4+ T cell count is typically $<100$ cell/pl (15). Within these patients, concurrent cryptococcosis, tuberculosis, Pneumocystis jirovecii pneumonia or concurrent cytomegalovirus and salmonella infections, are common (6-9). However, concurrent infection with T. marneffei and C. neoformans are rarely reported. In the current case report, the literature was reviewed and 8 cases of concurrent infection with T. marneffei and $C$. neoformans were presented in patients who were HIV-positive (7). To the best of our knowledge, this is the first case report describing a patient with concurrent infection with T. marneffei and C. neoformans who was also HIV-negative. The two types of fungi were simultaneously identified from blood culture (T. marneffei and C.neoformans) and bronchial mucosal biopsy cultures (C. neoformans).

Immunodeficiency represents a major risk factor for T. marneffei infection (5). Ma et al (16) impaired the immune state of monocytes via the addition of dexamethasone and declared that immunosuppressants, including dexamethasone, exhibit inhibitory effects on monocytes and macrophages, and can increase patient susceptibility to T. marneffei infection. CD4+ T cell-induced immunodeficiency may also serve a pathogenic role in HIV-negative patients with immunodeficiency $(2,13,17)$. A number of reports have indicated that anti-cytokine and anti-interferon- $\gamma$ autoantibodies are associated with adult-onset immunodeficiency $(1,14,17,18)$. These results indicated another possible reason for cellular immunodeficiency in patients who are HIV-negative, which contributes to host susceptibility to T. marneffei infection. The patient presented in the current case study received long-term glucocorticoid treatment (oral dexamethasone) for erythroblastic anemia for 8 years. The resulting decrease in white blood cell count further led to immunosuppression and T-cell immunodeficiency. Therefore, the patient exhibited an increased susceptibility to opportunistic infections. Unfortunately, the detection techniques were not available in the hospital this patient was admitted to, so these autoantibodies (including anti-cytokine and anti-interferon- $\gamma$ autoantibodies) were not detected, which is a limitation of the current case study.

Talaromycosis isassociated withnon-specific manifestations, including fever, weight loss, systemic lymph node enlargement, hepatosplenomegaly, skin lesions and anemia $(4,19)$. Evidence has indicated that symptoms of T. marneffe $i$ infection in patients who are HIV-negative differ from those in patients who are HIV-positive due to the disparities in the causes and features of immunosuppression $(5,15)$. The patient of the current case study presented with fever, cough, lymph node enlargement, anemia and thrombocytopenia, all of which were non-specific, 


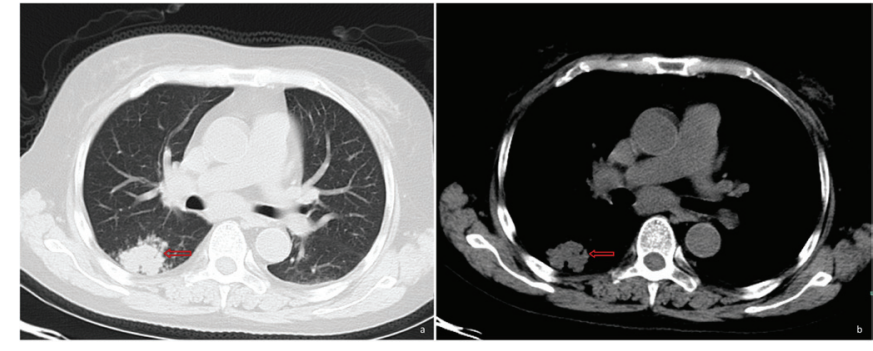

Figure 1. Pulmonary CT scan revealed a hyperdense mass (as indicated by the arrow) in the right lower lung, with unclear boundaries and non-uniform density.

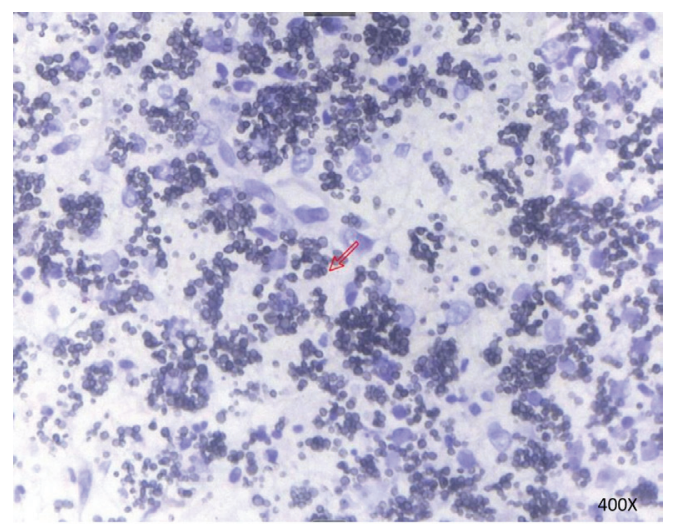

Figure 2. Sausage-like bacteria indicated by methenamine silver staining of the supraclavicular lymph nodes with septa. Talaromyces marneffei infection was indicated. The arrow indicates the septa. Magnification, x400.

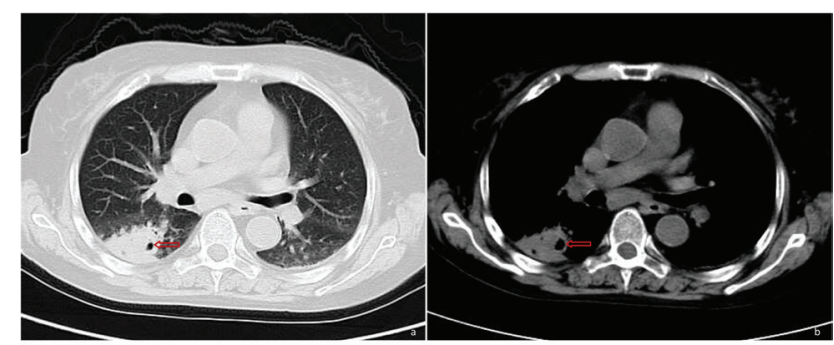

Figure 3. Solid lesion combined with cavitation (as indicated by the arrow) in the right lower lung by pulmonary CT.

and may lead to a misdiagnosis of tuberculosis or other fungal infections. The clinical implication of a misdiagnosis is that fungal infections are likely in patients with persistent fever following routine antibacterial therapy, with long-term glucocorticoid treatment or with an immunodeficiency disorder, and in these patients, concurrent opportunistic infections should be considered. Clinically, T. marneffei infection shares similar manifestations with tuberculosis or infections that are caused by $C$. neoformans, Histoplasma capsulatum and Nocadias. Concurrent infections with two or more pathogens should therefore be considered and confirmed by further tests. Furthermore, rare forms of these diseases deserve additional attention. The diagnosis and treatment of disseminated T. marneffei is difficult due to the existence of mixed infections, particularly in patients with concurrent fungal infections. Kawila et al (5) reported that T. marneffei infection-associated

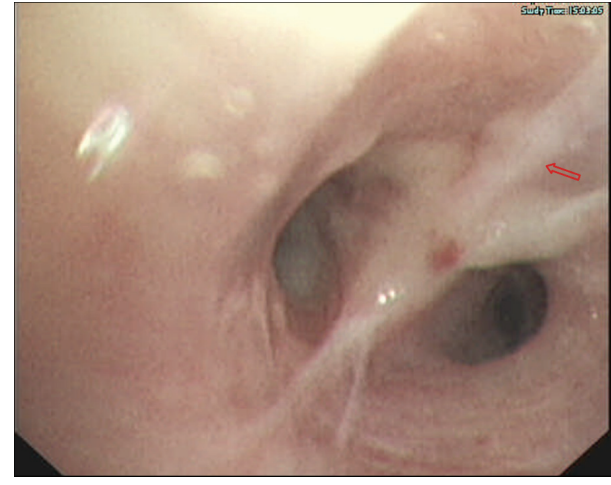

Figure 4. Bronchial mucosal edema and hyperplasia (as indicated by the arrow) indicated via bronchoscopy.

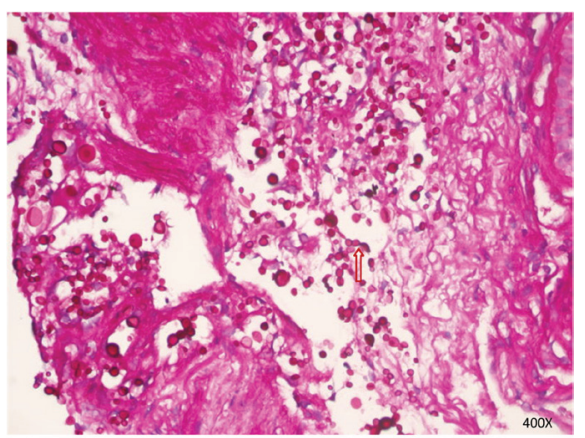

Figure 5. Cryptococcus neoformans spores (as indicated by the arrow) in bronchial mucosal mesenchyme indicated viaperiodic acid-Schiff staining. Magnification, $\mathrm{x} 400$.
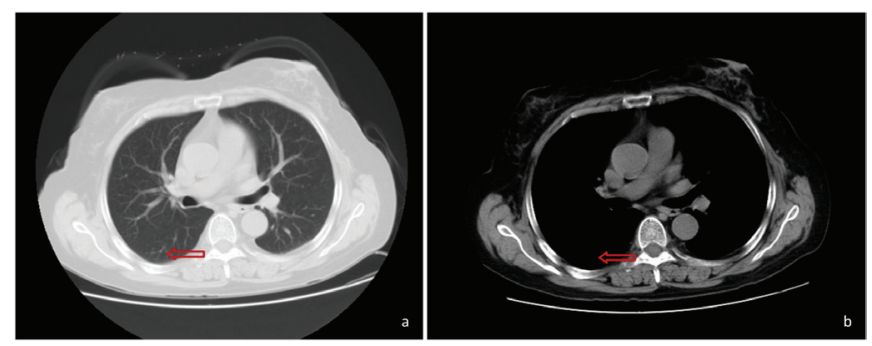

Figure 6. A follow-up chest CT scan revealed that the pulmonary lesion was absorbed (as indicated by the arrow) after the patient had been taking voriconazole tablets of $200 \mathrm{mg}$ twice a day for 2 months.

mortality in patients who were HIV-negative was unexpectedly higher than that inpatients infected with HIV. This result may be due to the fact that $T$. marneffei infection develops more quickly and a skin rash usually appears in patients who are HIV-positive, which makes diagnosis easier. In contrast, the symptoms of T. marneffei infection are more complex and more likely to be confused with other conditions in patients without HIV infection. Furthermore, mixed infections are common in patients without HIV infection, which contributes to a higher mortality (16). The patients presented in the current case study received intravenous voriconazole immediately following the diagnosis of T. marneffei infection. The symptoms of fever, cough, pharyngalgia and hemoptysis were improved gradually following treatment. The patient was changed to oral therapy 14 days after the treatment began. Blood cultures taken on 
day 5 indicated no T. marneffei growth, but indicated the growth of $C$. neoformans. These results demonstrated that the treatment was effective for T. marneffei infection, but unfortunately, it was undetermined as to whether $C$. neoformans infection occurred before or after the patient started receiving voriconazole. This was due to the fact no assessment of C.neoformans antigens or bronchoscopy was performed at the early stage of the disease and the repeat chest CT scan was not performed early enough. $C$. neoformans was simultaneously identified from serial blood cultures and confirmed using bronchial mucosal biopsy. The $C$. neoformans infection in the patient of the case study was sensitive to voriconazole, which was used to treat the T. marneffe $i$ infection, making it easier to effectively treat the patient and preventing the condition from becoming life threatening. The valuable clinical information demonstrated from the present case study is that the symptoms induced by the two pathogens in a concurrent infection may be similar. If a patient responds poorly to initial monotherapy, the possibility of coinfection should be considered. When coinfection occurs, it is necessary to determine if initial therapy is effective for treating all concurrent infections. If not, new treatment options should be considered. The patient of the current report did not exhibit any symptoms of central nervous system disorders and refused to receive a lumbar puncture. Due to this, whether the patient had $C$. neoformans meningitis could not be determined and this is a limitation of the current case report.

No standard antifungal treatment for T. Marneffei infection has been established and currently certain studies $(20,21)$ have identified that amphotericin B liposomal was effective in the treatment of T. Marneffei infection and recommended as initial therapy for the disease. However, amphotericin B's common side effects include electrolyte disturbance, renal compromise and hepatic impairment, which limits its clinical use (22). Voriconazole is an antifungal medication. It is in the triazole family of medications. Voriconazole has been revealed to exhibit potent in vitro activity and is used to treat multiple aggressive fungal infections with adequate safety and efficacy profiles (23). A recent retrospective study indicated that the intravenous administration of voriconazole as initial therapy for T. Marneffei infection was effective and well tolerated (24). The continuous administration of voriconazole for 12 weeks was demonstrated to be effective for the treatment of T. marneffei infection in patients with advanced HIV disease (25). However, no recommendation has been published on the duration of voriconazole administration for the treatment of disseminated T. marneffei infection in patients who are HIV-negative. A previous retrospective study indicated that the duration of therapy for T. marneffei infection was prolonged in patients who are HIV-negative compared with the treatment duration in patients who are HIV-positive (5). Voriconazole was used instead of amphotericin B for the current case report, following the confirmation of $T$. marneffei infection as Voriconazole has fewer side effects, is cheaper, was readily available at the hospital of admittance, is easier to purchase and has been determined to be effective in the treatment for talaromycosis $(22,24)$. A number of studies have indicated that C. neoformans was sensitive to voriconazole treatment $(26,27)$ and that its MIC value was low, indicating that voriconazole may be appropriate for the treatment of $C$. neoformans infection. Therefore, the patient continued to receive voriconazole following the confirmation of $C$. neoformans infection. Symptoms subsequently improved and the pulmonary lesion almost disappeared following treatment. For T. marneffei infection in patients who are HIV-negative, further studies are required to understand the duration that voriconazole should be administered, particularly in cases of concurrent fungal infections. The current case report should serve to indicate the importance of monitoring the condition of patients closely, to determine the most appropriate treatment duration of voriconazole. If no improvement is observed despite treatment at a dosage determined via drug susceptibility testing, prolonging initial treatment may be the most appropriate option.

In conclusion, the current case study indicated that despite being rare, concurrent septicemia and bronchopulmonary infection caused by $T$. marneffei and $C$. neoformans can occur. The risk of concurrent infection with $T$. marneffei and $C$. neoformans is higher in patients with a severely compromised immune system. When coinfection occurs, one pathogen may outgrow the other in blood cultures, masking the presence of the other therefore the diagnosis and treatment of concurrent infections with T. marneffe $i$ and $C$. neoformans are difficult. Clinicians should therefore be fully aware of the possibility of concurrent infections with T. marneffe $i$ and other opportunistic pathogens. For immunosuppressed patients, early diagnosis and treatment are crucial for improving patient prognosis. Further studies are warranted to better understand why an increasing number of patients who are HIV-negative are infected with T. marneffei or other opportunistic pathogens.

\section{Acknowledgements}

Not applicable.

\section{Funding}

Funding was received from The Science and Technology Foundation of Taizhou (grant no. 1801KY18) and the Projects of Medical and Health Technology Program in Zhejiang Province (grant no. 2015KYB439).

\section{Availability of data and materials}

The datasets used and/or analyzed during the present study are available from the corresponding author on reasonable request.

\section{Authors' contributions}

SH and XW conceived and designed the review. DL and YX prepared the patient data and figures. LL analyzed and interpreted the data. All authors agree to be accountable for all aspects of the research in ensuring that the accuracy or integrity of any part of the work are appropriately investigated and resolved. All authors read and approved the final manuscript.

\section{Ethics approval and consent to participate}

The Ethics Committee of Taizhou Hospital of Wenzhou Medical University passed the ethical review (approval no: K20190214). The Ethics Committee of Taizhou Hospital of Wenzhou Medical University waived the need for consent 
for the publication of patient information in the present manuscript due to the reason that the patient had passed away.

\section{Patient consent for publication}

Due to the death of the patient, no consent for publication was obtained.

\section{Competing interests}

The authors declare that they have no competing interests.

\section{References}

1. DiSalvo AF, Fickling AM and Ajello L: Infection caused by Penicillium marneffei: Description of first natural infection in man. Am J Clin Pathol 60: 259-263, 1973.

2. Vanittanakom N, Cooper CR Jr, Fisher MC and Sirisanthana T: Penicillium marneffei infection and recent advances in the epidemiology and molecular biology aspects. Clin Microbiol Rev 19 95-110, 2006

3. Ustianowski AP, Sieu TP and Day JN: Penicillium marneffei Infection in HIV. Curr Opin Infect Dis 21: 31-36, 2008.

4. Supparatpinyo K, Khamwan C, Baosoung V, Nelson KE and Sirisanthana T: Disseminated Penicillium marneffei infection in Southeast Asia. Lancet 344: 110-113, 1994.

5. Kawila R, Chaiwarith R and Supparatpinyo K: Clinical and laboratory characteristics of penicilliosis marneffei among patients with and without HIV infection in northern Thailand: A retrospective study. BMC Infect Dis 13: 464, 2013.

6. Zheng J, Gui X, Cao Q, Yang R, Yan Y, Deng L and Lio J: A clinical study of acquired immunodeficiency syndrome associated Penicillium mameffei infection from a non-endemic area in China. PLOS One 10: e0130376, 2015.

7. Le T, Hong Chau TT, Kim Cuc NT, Si Lam P, Manh Sieu TP, Shikuma CM and Day JN: AIDS-Associated Cryptococcus neoformans and Penicillium marneffei Coinfection: A therapeutic dilemma in resource-limited settings. Clin Infect Dis 51 e65-e68, 2010.

8. Larsson M, Nguyen LH, Wertheim HF, Dao TT, Taylor W, Horby P, Nguyen TV, Nguyen MH, Le T and Nguyen KV: Clinical characteristics and outcome of Penicillium mameffei infection among HIV-infected patients in northern Vietnam. AIDS Res Ther 9: 24, 2012.

9. Hatakeyama S, Yamashita T, Sakai T and Kamei K: Case Report: Disseminated Talaromyces (Penicillium) marneffei and mycobacterium tuberculosis coinfection in a japanese patient with acquired immunodeficiency syndrome. Am J Trop Med Hyg 97: 38-41, 2017

10. Nascimento E, Vitali LH, Kress MRVZ and Martinez R: Cryptococcus neoformans and C. Gattii isolates from both HIV-infected and uninfected patients: Antifungal susceptibility and outcome of cryptococcal disease. Rev Inst Med Trop Sao Paulo 59: e49, 2017.

11. Lin JN, Lin HH, Lai CH, Wang JL and Yu TJ: Renal transplant recipient infected with Penicillium marneffei. Lancet Infect Dis 10: 138, 2010.

12. Chong YB, Tan LP, Robinson S, Lim SK, Ng KP, Keng TC and Kamarulzaman A: Penicilliosis in lupus patients presenting with unresolved fever: A report of 2 cases and literature review. Trop Biomed 29: 270-276, 2012 .
13. Lee PP, Chan KW, Lee TL, Ho MH, Chen XY, Li CH, Chu KM, Zeng HS and Lau YL: Penicilliosis in children without HIV infection-are they immunodeficient? Clin Infect Dis 54: e8-e19, 2012.

14. Chitasombat M and Supparatpinyo K: Penicillium marneffei infection in immunocompromised host. Curr Fungal Infect Rep 7: 44-50, 2013.

15. Wong SY and Wong KF. Penicillium mameffei infection in AIDS. Pathol Res Int 2011: 764293, 2011.

16. Ma T, Chen R, Li X, Lu C and Xi L: The in vitro fungicidal activity of human macrophages against Penicillium marneffei is suppressed by dexamethasone. Microb Pathog 86: 26-31, 2015.

17. Browne SK, Burbelo PD, Chetchotisakd P, Suputtamongkol Y, Kiertiburanakul S, Shaw PA, Kirk JL, Jutivorakool K, Zaman R, Ding L, et al: Adult-onset immunodeficiency in thailand and taiwan. N Engl J Med 367: 725-734, 2012.

18. Lee PP, Mao H, Yang W, Chan KW, Ho MH, Lee TL, Chan JF, Woo PC, Tu W and Lau YL: Penicillium marneffei infection and impaired IFN- $\gamma$ immunity in humans with autosomal-dominant gain-of-phosphorylation STAT1 mutations. J Allergy Clin Immunol 133: 894-896.e5, 2014.

19. Vanittanakom N and Sirisanthana T: Penicillium marneffei infection in patients infected with human immunodeficiency virus. Curr Top Med Mycol 8: 35-42, 1997.

20. Sirisanthana T, Supparatpinyo K, Perriens J and Nelson KE: Amphotericin B and itraconazole for treatment of disseminated Penicillium marneffei infection in human immunodeficiency virus-infected patients. Clin Infect Dis 26: 1107-1110, 1998 .

21. Wang JL, Hung CC, Chang SC, Chueh SC and La MK: Disseminated Penicillium marneffei infection in a renal-transplant recipient successfully treated with liposomal amphotericin B. Transplantation 76: 1136-1137, 2003.

22. Walsh TJ, Pappas P, Winston DJ, Lazarus HM, Petersen F, Raffalli J, Yanovich S, Stiff P, Greenberg R, Donowitz G, et al: Voriconazole compared with liposomal amphotericin B for empirical antifungal therapy in patients with neutropenia and persistent fever. N Engl J Med 346: 225-234, 2002.

23. Zmeili OS and Soubani AO: Pulmonary aspergillosis: A clinical update. QJM 100: 317-334, 2007.

24. Ouyang Y, Cai S, Liang H and Cao C: Administration of voriconazole in disseminated talaromyces (Penicillium) Marneffei Infection: A Retrospective Study. Mycopathologia 182: 569-575, 2017.

25. Perfect JR, Marr KA, Walsh TJ, Greenberg RN, DuPont B, de la Torre-Cisneros J, Just-Nübling G, Schlamm HT, Lutsar I, Espinel-Ingroff A and Johnson E: Voriconazole treatment for less-common, emerging, or refractory fungal infections. Clin Infect Dis 36: 1122-1131, 2003

26. Alves IA, Staudt KJ, Silva CM, Lock GA, Dalla Costa T and de Araujo BV: Influence of experimental cryptococcal meningitis in wistar rats on voriconazole brain penetration assessed by microdialysis. Antimicrob Agents Chemother 61: pii: e00321-e00417, 2017.

27. Herkert PF, Hagen F, de Oliveira Salvador GL, Gomes RR, Ferreira MS, Vicente VA, Muro MD, Pinheiro RL, Meis JF and Queiroz-Telles F: Molecular characterisation and antifungal susceptibility of clinical Cryptococcus deuterogattii (AFLP6/VGII) isolates from Southern Brazil. Eur J Clin Microbiol Infect Dis 35: 1803-1810, 2016.

This work is licensed under a Creative Commons Attribution-NonCommercial-NoDerivatives 4.0 International (CC BY-NC-ND 4.0) License. 